\title{
Structural and Optical Properties of
}

\section{$\mathrm{Cs}_{2} \mathrm{AgBiBr}_{6}$ Double Perovskite.}

\author{
Laura Schade, ${ }^{*}$ Adam D. Wright, Roger D. Johnson, Markus Dollmann, Bernard \\ Wenger, Pabitra K. Nayak, Dharmalingam Prabhakaran, Laura M. Herz, Robin \\ Nicholas, Henry J. Snaith, and Paolo G. Radaelli \\ Clarendon Laboratory, Department of Physics, University of Oxford, OX1 3 PU, United \\ Kingdom. \\ E-mail: laura.schade@physics.ox.ac.uk.
}

\begin{abstract}
We present a comprehensive study of the relationship between the crystal structure and optoelectronic properties of the double perovskite $\mathrm{Cs}_{2} \mathrm{AgBiBr}_{6}$, which has emerged as a promising candidate for photovoltaic. Based on single-crystal/powder X-ray diffraction and neutron powder diffraction, we have revealed the presence of a structural phase transition at $\mathrm{T}_{s} \sim 122 \mathrm{~K}$ between the room-temperature cubic structure (space group $F m \overline{3} m$ ) and a new low-temperature tetragonal structure $(I 4 / m)$. From reflectivity measurements we found that the peak exciton energy $E_{e x} \approx 2.85 \mathrm{eV}$ near the direct gap shifts proportionally to the tetragonal strain, which is consistent with the $\mathrm{E}_{\text {ex }}$ being primarily controlled by a rotational degree of freedom of the crystal structure, thus by the angle $B i-B r-A g$. We observed the time-resolved photoluminescence kinetics and we found that, among the relaxation channels, a fast one is mainly present in the tetragonal phase, suggesting that its origin may lie in the formation of tetragonal twin domains.
\end{abstract}




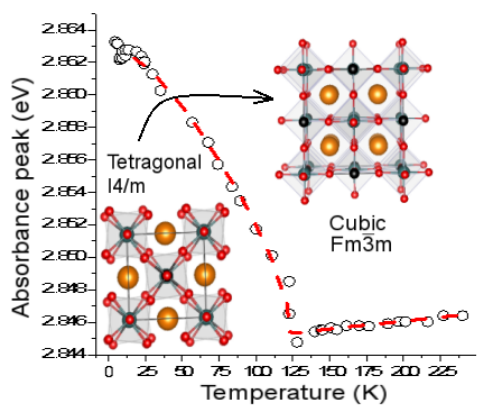

\section{Table of contents}

Hybrid halide perovskites, with general formula $\mathrm{ABX}_{3}(\mathrm{~A}=$ organic/inorganic $1+$ cation, $\mathrm{B}=$ inorganic $2+$ cation and $\mathrm{X}=$ halide anion) have gained increasing attention in the scientific community as high-performing semiconductors in solar cell devices. The Power Conversion Efficiency of devices based on these materials has increased to a remarkable $28 \%$ in the last few years, 1 due to high carrier mobility, a tunable band gap, long diffusion lengths, and strong optical absorption. ${ }^{2+5}$ For highest thermal stability, an all-inorganic perovskite would be preferable. However, to-date it has proven highly challenging to stabilise the room temperature crystalline polymorph of the inorganic lead halide $\mathrm{CsPbI}_{3} \cdot \stackrel{[6}{[}$ Furthermore, the realisation of the unexpected functionality of the lead halide perovskites has directed the research community to attempt to discover new metal halide based semiconductors with improved, or complementary, functionality.

Recently, double perovskites with general formula $\mathrm{A}_{2}^{1+} \mathrm{M}^{1+} \mathrm{M}^{3+} \mathrm{X}_{6}$ have been proposed as all-inorganic alternatives. In particular, $\mathrm{Cs}_{2} \mathrm{AgBiBr}_{6}$ is one of the few materials investigated since the discovery of efficient PV operation of lead halide perovskites which delivers substantial performance in a PV cell. It is highly stable, ${ }^{[7}$ it has been predicted to have relatively low carrier effective masses ${ }^{[8}$ and it has shown long carrier recombination lifetimes. ${ }^{9}$ Greul et al. ${ }^{10}$ and Gao et al. ${ }^{11}$ demonstrated the fabrication of $\mathrm{Cs}_{2} \mathrm{AgBiBr}_{6}$ films and incorporated them into working devices for the first time. However, there is very little know about the crystallography and its impact upon the optoelectronic properties. This information is important in order to both improve the present family of double perovskites, and to design 
new and improved compounds. Although the maximum power conversion efficiency so far achieved in double perovskites $(2.23 \%)$ is lower than for hybrid perovskites, these materials are much less mature in terms of device technologies and have significant potential for applications, as demonstrated for example by the discovery of highly efficient near white light emission from $\mathrm{Cl}$ double perovskites. $\frac{[12}{}$ Furthermore, they represent a very good model system to study, in order to understand deficiencies and hence routes to improved properties through either design of new compounds, or the tuning of the present ones.

Based on X-ray diffraction measurements, McClure et al. ${ }^{[13}$ determined the ambienttemperature crystal structure of $\mathrm{Cs}_{2} \mathrm{AgBiBr}_{6}$ to be cubic with $a=11.2711(1) \AA$ and space group $F m \overline{3} m$, which accounts for the $\mathrm{Bi} / \mathrm{Ag}$ ordering on a rock-salt superstructure. An indirect band gap has also been reported around $\sim 2 \mathrm{eV}$, but the community is yet to find consensus on its exact value. ${ }^{913114}$ Recently, the charge carrier dynamics of this compound has been explored with different techniques. ${ }^{[5116}$ In spite of these efforts, very little is known about the relationship between structural and optical properties, and in particular the direct band gap transition. Originally predicted to be at the $\mathrm{X}$-point and $0.7 \mathrm{eV}$ higher than the indirect gap, $\frac{14}{14}$ it is visible at $\sim 2.8 \mathrm{eV}$ as a sharp feature typical of two-particle transitions, and is the dominant feature in the absorbance and reflectivity of thin films, $\frac{16}{16}$ nanocrystals $\frac{17}{17}$ and bulk crystal reflectivity. ${ }^{13}$ Regardless of whether $\mathrm{Cs}_{2} \mathrm{AgBiBr}_{6}$ devices can be optimised, the remainder of this paper will demonstrate that $\mathrm{Cs}_{2} \mathrm{AgBiBr}_{6}$ is an extraordinary interesting model system for understanding the physics of the wider family of halide perovskites. All these materials have closely related crystal structures, and understanding structure-property relations is highly relevant for materials that are already known to have higher efficiency, and for guiding the design of future compounds. For instance, $\mathrm{MAPbI}_{3}$ undergoes ferroelastic phase transitions both above and below room temperature ${ }^{\frac{18}{18}}$ that are closely related to $\mathrm{Cs}_{2} \mathrm{AgBiBr}_{6}$ and crystallises in a distorted phase at operating temperatures. Therefore, rational design of better halide PV materials will be greatly facilitated by understanding structure-property relations in model systems such as $\mathrm{Cs}_{2} \mathrm{AgBiBr}_{6}$. 
In this paper, we describe the structural properties of $\mathrm{Cs}_{2} \mathrm{AgBiBr}_{6}$ as a function of temperature, and their correlation with its optoelectronic properties. Based on heat capacity measurements, X-ray powder and single-crystal diffraction and neutron powder diffraction, we demonstrate that this compound undergoes a low-temperature cubic to tetragonal structural phase transition at $\mathrm{T}_{S} \sim 122 \mathrm{~K}$. We refine the published cubic crystal structure above $\mathrm{T}_{S}$, and we present a complete structural solution for the low-temperature tetragonal phase. Having established the temperature dependence of the excitonic transition at the direct band gap from reflectivity measurements, we found a linear relationship with the tetragonal strain. This demonstrates that the direct gap is primarily controlled by $M \mathrm{Br}_{6}$ octahedral rotations that characterize the tetragonal phase. Finally, based on photoluminescence measurements, we evidence the presence of enhanced PL near the tetragonal phase transition, and, below it, of an additional fast charge-carrier recombination mechanism, which is suppressed in the cubic phase and may be associated with strain at tetragonal twin boundaries.

Polycrystalline powder samples were synthesized through precipitation: the chemicals $\mathrm{AgBr}$ (2mmol, 99.5\% of purity), $\mathrm{CsBr}$ (4mmol, 99\% of purity) and $\mathrm{BiBr}_{3}$ (2mmol, purity $\geqslant$ $98 \%$ ) were dissolved in $\sim 4 \mathrm{ml}$ of DMSO (dimethyl sulfoxide) and then precipitated with acetone, producing $\sim 2$ grams of powder after drying. Using commercial chemicals, we could not grow single crystals as previously reported ${ }^{9}$ due to the lack of complete solubility of the salts in hot $\mathrm{HBr}$ solution. So, we first precipitated the polycrystalline powder, and then we dissolved the compound $(\sim 1 \mathrm{~g})$ in $\sim 10 \mathrm{ml}$ of $9-\mathrm{M} \mathrm{HBr}$, heating it at $110^{\circ}$ in an oven. Cooling down to room temperature at a rate of 0.5 degrees per hour we obtained $\sim 1 \mathrm{~mm}-$ size single crystals. We characterized both single crystal and powder samples with the use of X-ray diffraction (see Section 7 in the Supplementary Information (SI) for experimental details). The single crystal data were fit with the established cubic crystal structure ${ }^{13}$ giving outstanding goodness-of-fit, which demonstrated excellent crystal quality (see Figure S1, S2 and table S1 in the SI). The X-ray powder diffraction data was also fit using the same cubic structure model, which well reproduced the majority of peaks in the diffraction pattern. 
However, a $20 \mathrm{wt} \% \mathrm{CsAgBr}_{2}$ impurity phase was also identified. In both refinements, the B-site cations were found to be fully ordered within the sensitivity of the measurement (the occupancy of both the atoms was found to be $1.00 \pm 0.05$ ). This is important to note since it has been predicted that the optical properties may change with the degree of occupational disorder of the B-site cations. $\frac{19}{19}$

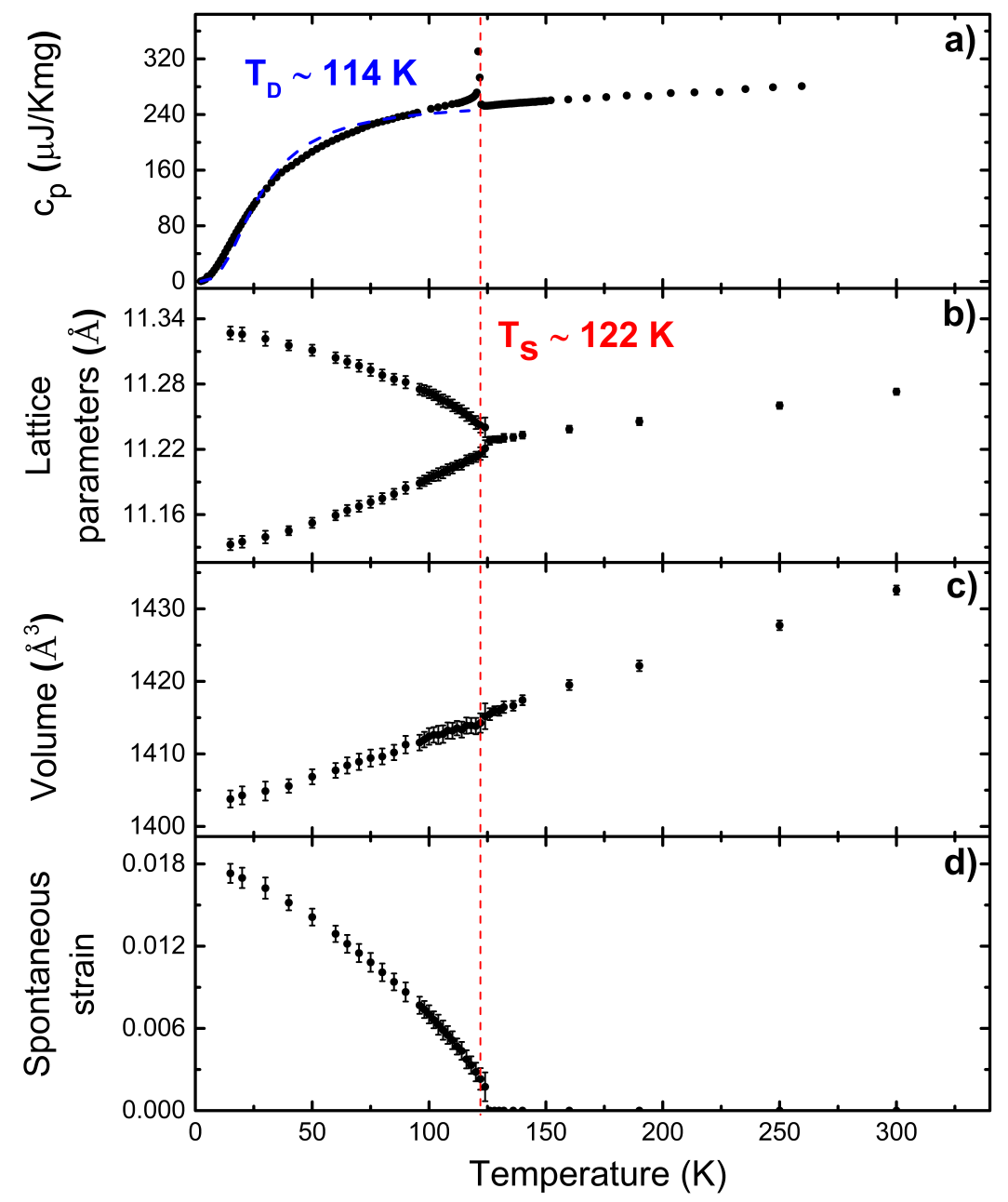

Figure 1: The temperature dependence of a) the heat capacity (error bars are smaller than the data points). The dashed line is a Debye fit to the data below the phase transition. b) lattice parameters, c) the unit cell volume, and d) the spontaneous strain. The red line marks the transition temperature at $\mathrm{T}_{s} \simeq 122 \mathrm{~K}$.

We performed specific heat capacity $\left(\mathrm{c}_{p}\right)$ measurements on a $5.2 \mathrm{mg}$ single crystal (Exper- 
imental details in the SI). We show the data in Figure $1 \mathrm{a}$, where we observe a sharp anomaly at $T_{s} \simeq 122 \mathrm{~K}$, indicative of a second-order or weakly first-order phase transition. Furthermore, from detailed $c_{p}$ measurements on heating and cooling near the transition temperature $\mathrm{T}_{s}$ (see Figure S5 in the SI) we observe the absence of thermal hysteresis. From the data in Figure 1 $1 \mathrm{a}$, we fit the $\mathrm{T}<T_{s} \mathrm{c}_{p}$ range with a Debye model, yielding a Debye temperature $T_{D}=114(4) \mathrm{K}$. This temperature is consistent with lower values of the sound velocity and bulk modulus than the most commonly known hybrid-halide perovskites ${ }^{20}$ suggesting that $\mathrm{Cs}_{2} \mathrm{AgBiBr}_{6}$ is slightly softer.

We confirmed the presence of a structural phase transition at $\mathrm{T}_{S}$ by powder XRD. Immediately below $\mathrm{T}_{S}$, diffraction peaks became strongly split (see Figure $\mathrm{S} 4$ in the SI), consistent with the lowering of the crystal symmetry to tetragonal with a unit-cell elongation along the $c$ axis. This symmetry then persists down to the lowest measured temperature (15K). Assuming tetragonal symmetry, we fitted the lattice parameters to the data, which we display in Figure 1 b).

In order to determine the low-temperature tetragonal crystal structure of $\mathrm{Cs}_{2} \mathrm{AgBiBr}_{6}$ accurately, we collected neutron powder diffraction (NPD) data at room temperature and at $30 \mathrm{~K}$ (see Experimental details in SI). To check for consistency, we refined the cubic structure $^{13}$ against our room-temperature NPD data (Figure S3 a in the SI) and we found it in excellent agreement with our previous refinement using single-crystal XRD. We list the two sets of room-temperature structural parameters in Table I.

Symmetry analysis was performed using the Isotropy Suite. ${ }^{21}$ There are just two candidate tetragonal subgroups of the $F m \overline{3} m$ parent structure: $I 4 / m m m$ (no. 189) is reached by a tetragonal distortion of the $M \mathrm{Br}_{6}$ octahedra. Alternatively, subgroup $I 4 / m$ (no. 87 ) also allows for a coherent rotation of $\mathrm{MBr}_{6}$ octahedra about the tetragonal c axis, where $\mathrm{BiBr}_{6}$ and $\mathrm{AgBr}_{6}$ octahedra rotate in opposite senses (see Figure S2 in the SI). Since $I 4 / m$ is a subgroup of $I 4 / \mathrm{mmm}$, the atomic displacements allowed in $I 4 / \mathrm{mmm}$ are also allowed in $I 4 / m$; hence, we can refine the crystal structure using the $I 4 / m$ space group, and assess $a$ 
Table 1: Refined crystal structure parameters of $\mathrm{Cs}_{2} \operatorname{AgBiBr}_{6} . \mathrm{a}_{t}=\frac{1}{2} \mathrm{a}_{c}+\frac{1}{2} \mathrm{~b}_{c} ; \mathrm{b}_{t}=-\frac{1}{2} \mathrm{a}_{c}+\frac{1}{2} \mathrm{~b}_{c}$, where subscripts $\mathrm{c}$ and $\mathrm{t}$ refer to the cubic and tetragonal unit cells, respectively. In $F m \overline{3} m$ the atoms are located at the following Wyckoff positions. Cs: 8c $\left(\frac{1}{4}, \frac{1}{4}, \frac{1}{4}\right)$; $\mathrm{Bi}$ : $4 \mathrm{a}(0,0$, $0)$; $\mathrm{Ag}: 4 \mathrm{~b}\left(\frac{1}{2}, \frac{1}{2}, \frac{1}{2}\right)$; Br1: $24 \mathrm{e}(\mathrm{x}, 0,0)$. In the $I 4 / m$ space group: Cs: $4 \mathrm{~d}\left(\frac{1}{2}, 0, \frac{1}{4}\right) ; \mathrm{Bi}: 2 \mathrm{a}$ $(0,0,0)$; Ag: $2 \mathrm{~b}\left(0,0, \frac{1}{2}\right) ; \operatorname{Br} 1: 8 \mathrm{~h}(\mathrm{x}, \mathrm{y}, 0) ; \mathrm{Br} 2: 4 \mathrm{e}(0,0, \mathrm{z}) . \mathrm{V}_{f . u}$ stands for volume per formula unit.

\begin{tabular}{c|ccc} 
& NPD 30K & NPD RT & PXRD RT \\
\multicolumn{5}{|c}{$I 4 / m$} & $F m \overline{3} m$ & $F m \overline{3} m$ \\
Lattice Parameters $[\AA]$ & & \\
$a_{t, c}$ & $7.8794(4)$ & $11.2784(5)$ & $11.27301(9)$ \\
$c_{t, c}$ & $11.3236(7)$ & $11.2784(5)$ & $11.27301(9)$ \\
$V_{f . u .}\left[\AA^{3}\right]$ & $351.51(3)$ & $358.66(3)$ & $358.145(5)$ \\
Refinable atomic fractional coordinates \\
Br1 $x$ & $0.27976(2)$ & $0.25072(1)$ & $0.25143(2)$ \\
$y$ & $0.77941(2)$ & - & - \\
Br2 $z$ & $0.24959(2)$ & - & - \\
Atomic displacement & parameters & $\mathbf{1 0 0} \times \mathbf{U}_{i s o}\left[\AA^{2}\right]$ \\
Cs & $0.900(9)$ & $4.517(1)$ & $3.56(4)$ \\
Ag & $0.204(5)$ & $1.169(1)$ & $0.55(4)$ \\
Bi & $0.475(5)$ & $2.229(2)$ & $1.99(8)$ \\
Br1 & $0.966(7)$ & $4.879(1)$ & $4.05(4)$ \\
Br2 & $0.966(7)$ & - & - \\
Refinement reliability & parameters $[\%]$ \\
$R_{p}$ & 1.02 & 1.37 & 0.96 \\
$R_{w p}$ & 1.04 & 1.41 & 2.59
\end{tabular}

posteriori whether the additional symmetry lowering is justified. An $I 4 / m$ crystal structure model was Rietveld-refined against neutron powder diffraction data collected at $30 \mathrm{~K}$, giving excellent agreement (see Figure S3 b) and Table I). With respect to the cubic phase, the octahedra underwent a tetragonal distortion of $\mathrm{r}_{z} / \mathrm{r}_{x y}=1.009 \pm 0.008$ for the $\mathrm{AgBr}_{6}$ one and $1.007 \pm 0.009$ for the $\mathrm{BiBr}_{6}$ one (where $\mathrm{r}_{x y}$ and $\mathrm{r}_{z}$ represent the bond lengths between $\mathrm{Ag} / \mathrm{Bi}$ and $\mathrm{Br}$ in the plane and along the $\mathrm{c}$ axis respectively). On the other hand, the octahedra in the tetragonal phase rotate by $\theta=6.75^{\circ} \pm 0.15$ (see Figure S2 in the SI). Based on these results, it is clear that the primary distortion below $\mathrm{T}_{S}$ is the octahedral rotations and the tetragonal elongation is negligible, further confirming the choice of the $I 4 / \mathrm{m}$ space group.

The $F m \overline{3} m \rightarrow I 4 / m$ phase transition is allowed to be second-order, and its order parameter $\eta$ is proportional to the octahedral rotation angle, while the spontaneous tetragonal 
strain $\sigma=2(a-c) /(a+c)$ is proportional to $\eta^{2}$. It is important to note that $\eta$ is here related to the rotational degree of freedom, and it should not be confused with any order parameter associated with a transition driven by the disorder on the B-site cations as discussed previously. 19$]$ In the mean-field approximation, $\eta^{2}$ should be linearly proportional to the reduced temperature $t=1-T / T_{s}$. More generally, the order parameter should follow the relation $\eta=t^{\beta}$, with the critical exponent $\beta=1 / 2$ in mean field.

The tetragonal strain (shown in Figure 1 d) obeys a linear relationship reasonably well near the phase transition, whilst departing from it markedly at low temperatures; it is in general very difficult to determine $\beta$ with any accuracy, and even the possibility of a weakly first-order transition (always allowed) cannot be completely excluded.

From reflectivity measurements (Experimental details in the SI) performed on a single crystal, we observe that the predominant feature in the spectrum is a dispersive edge, characteristic of a band-edge, free-carrier excitonic absorption, which strengthens rapidly and narrows with decreasing temperature (see Figure S7). Using an approximation of the Kramers-Kronig relations, the negative energy derivative of the reflectivity $(-d R / d E)$, produces peaks at almost exactly the energies of the resonant absorption and improves the spectral resolution (see SI for a full analysis). Figure 2 a demonstrates the narrowing and shift with temperature. By $1.5 \mathrm{~K}$ the full width at half maximum is only $30 \mathrm{meV}$ (corresponding to a spectral broadening of $60 \mathrm{meV}$ ) allowing much greater accuracy in measurement of the interband excitonic transition energy $E_{\text {ex }}$. In Figure $2 \mathbf{b}$ we show the temperature dependence of the absorbance peak values (open circles). The dashed line is a model constructed based on the strain data in Figure $1 \mathrm{~d}$ and assuming the following functional relation:

$$
E_{\text {ex }}=E_{\text {gap }}-E_{\mathrm{b}}=c_{0}+c_{1} \frac{\Delta V}{V_{0}}+c_{2} \sigma
$$

where $E_{\text {ex }}$ is the exciton energy, $E_{\text {gap }}$ is the direct energy gap, $E_{\mathrm{b}}$ is the exciton binding energy, $V$ is the unit cell volume, and $\sigma$ is the spontaneous strain, derived from the data in Figure 1 b. The model, in excellent agreement with the data, was constructed by assuming 

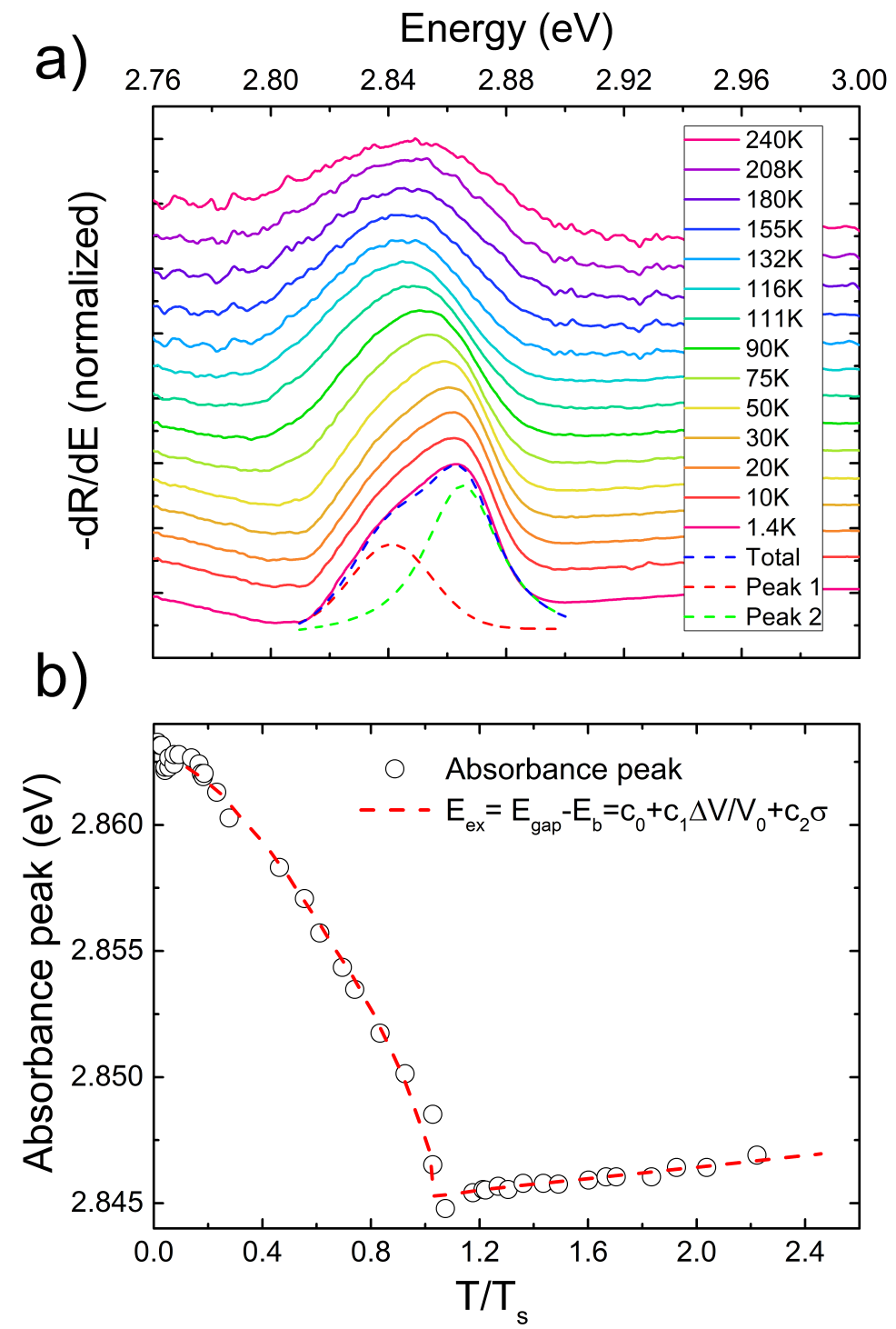

Figure 2: (a) Temperature dependence of normalized differential reflectivity (-dR/dE). The data are scaled for a better visualization. The lowest temperature spectrum has been fitted with two peaks shown by the dashed lines, which have the same linewidth and an intensity ratio of 2:1 and a splitting of $25 \mathrm{meV}$. (b) Absorbance peaks values (empty circles) plotted as a function of the reduced temperature and fitted with eq. 1 (dashed line). The fit demonstrates the linear relationship between the increase in exciton energy in the tetragonal phase and the tetragonal strain.

that $E_{\mathrm{b}}$ is independent of temperature, while $E_{\text {gap }}$ is controlled by and proportional to the electronic bandwidth $W$ in the temperature range we probed. In this framework, there are two parameters controlling the bandwidth: the M-X bond length $d$, which increases 
monotonically with temperature, and the M-X-M' bond angle $\theta$, which is related to the octahedral rotation (the order parameter $\eta$ ) as $\theta+2 \eta=\pi$. By denoting $\Delta E_{g}$ as the change in gap energy from the a hypothetical undistorted perovskite $(\theta=\pi), d_{0}$ and $V_{0}$ as the zero-temperature M-X bond length and cell volume respectively, and $\gamma$ as the appropriate exponent for the bond length, we obtain:

$$
\begin{aligned}
\Delta E_{g}= & \tilde{c}_{1}\left(d^{\gamma}-d_{0}^{\gamma}\right)+\tilde{c}_{2}(\cos \theta+1) \approx \\
& \tilde{c}_{1} d_{0}^{\gamma} \frac{\gamma}{3} \frac{\Delta V}{V_{0}}+2 \tilde{c}_{2} \eta^{2}
\end{aligned}
$$

which is equivalent to Equation 11. It is evident from Figure $2 \mathbf{b}$ that, for $\mathrm{Cs}_{2} \mathrm{AgBiBr}_{6}$, changes in the exciton energy are largely dominated by bond angles rather than bond distances. In fact, our determinated value for $c_{1} \sim 0.16 \mathrm{eV}$ is small in comparison with $c_{2}=$ $1.01 \pm 0.02 \mathrm{eV}$. A change in band gap of $\sim 1 \mathrm{eV}$ has also been observed by Stoumpos and Kanatzidis ${ }^{22}$ for the family of $A \mathrm{PbI}_{3}$ compounds where $\theta$ varies from $\pi$ to $\pi / 2$ in a sequence of different crystal structures, while the dominant role of the M-X-M angle on the bandgap changes has been discussed for the tin-iodide perovskites. ${ }^{23}$ At the lowest temperatures, the spectra in Fig. 2 a suggest that there is an unresolved splitting of $\sim 25$ meV into two peaks with an intensity ratio of $2: 1$, consistent with a strain induced lifting of the $\mathrm{X}$ band gap degeneracy.

We performed steady state photoluminescence (PL) measurements, collected at room and at low temperatures with an excitation wavelength of $398 \mathrm{~nm}$ (see the SI for details of the experimental set-up). At $25 \mathrm{~K}$ we observe the presence of a PL peak at $\left(\mathrm{E}_{\mathrm{PL}}\right)$ at $\sim 1.97(1) \mathrm{eV}$, i.e., $\sim 0.9 \mathrm{eV}$ below the exciton energy, with a FWHM of $\sim 0.2 \mathrm{eV}$. We only observe a weak dependence of the PL peak position with increasing temperature, while the peak width increases to $\sim 0.6 \mathrm{eV}$ at room temperature. The values of the peak maxima correlate well with our absorption measurements (Figure 3, panels a and b). We fit the absorption 
spectrum accurately to the functional form $\propto\left(E-E_{g}^{i}\right)^{2}$, which is consistent with an indirect bandgap ${ }^{24}$ whose onset correlates well with the values of the PL peak maxima. This agrees with the consensus $\frac{14116}{1}$ that the PL can be assigned to recombination across the indirect bandgap, although a defect-assisted recombination pathway has also been proposed. $\frac{15}{15}$ The value of the indirect gap at room temperature $(1.87(3) \mathrm{eV})$ is within the range of those previously reported in the literature (between $1.83 \mathrm{eV}$ and $2.25 \mathrm{eV}), 25$ and increases to $~$ $1.97 \mathrm{eV}$ at $25 \mathrm{~K}$. We note that this direction of band gap change with temperature is opposite to that usually observed for lead and tin halide perovskites, but the same as that observed for most conventional semiconductors, such as Si and GaAs. 26

Time-dependent PL measurements collected at $\mathrm{T}=4 \mathrm{~K}$ (Figure 3, panel c) indicate the presence of two different channels for PL decay: the first component, labelled S for 'short', was modelled with a mono-exponential, and has a very short time constant of $\tau_{\text {short }} \sim 1 \mathrm{~ns}$, while the second, labelled L has a very long time constant, such that it cannot be reliably measured at this temperature. As shown in Figure $3 \mathbf{c}$, on warming the lifetime of the $\mathrm{S}$ component remains much lower than that of the L component, while its overall intensity decreases. By contrast, the decay of the L component becomes faster on warming. Above $50 \mathrm{~K}$, the L component can be fitted with a stretched exponential, yielding average lifetimes that vary between $\sim 50 \mu \mathrm{s}$ at $50 \mathrm{~K}$ to a few ns at $200 \mathrm{~K}$ and above (panel $\mathbf{c}$ and $\mathbf{d}$ ).

We show the intensity ratio between the $\mathrm{S}$ and $\mathrm{L}$ components in an inset of Figure 3 . panel e). When correlated with the structural and reflectivity measurements, these data demonstrate that the $\mathrm{S}$ decay channel is only present in the tetragonal phase, and that it rapidly becomes weaker as the tetragonal distortion is reduced. One intriguing possibility is that $\mathrm{S}$ could be associated with defects concentrating at the twin boundaries, which spontaneously form on cooling through the phase transition. In this scenario, the onset of the S component need not correlate precisely with $\mathrm{T}_{S}$, because a minimum value of twin boundary strain is usually required for the formation of defects. This hypothesis could be verified by repeating the PL measurements on a single crystal cooled under biaxial compressive strain 

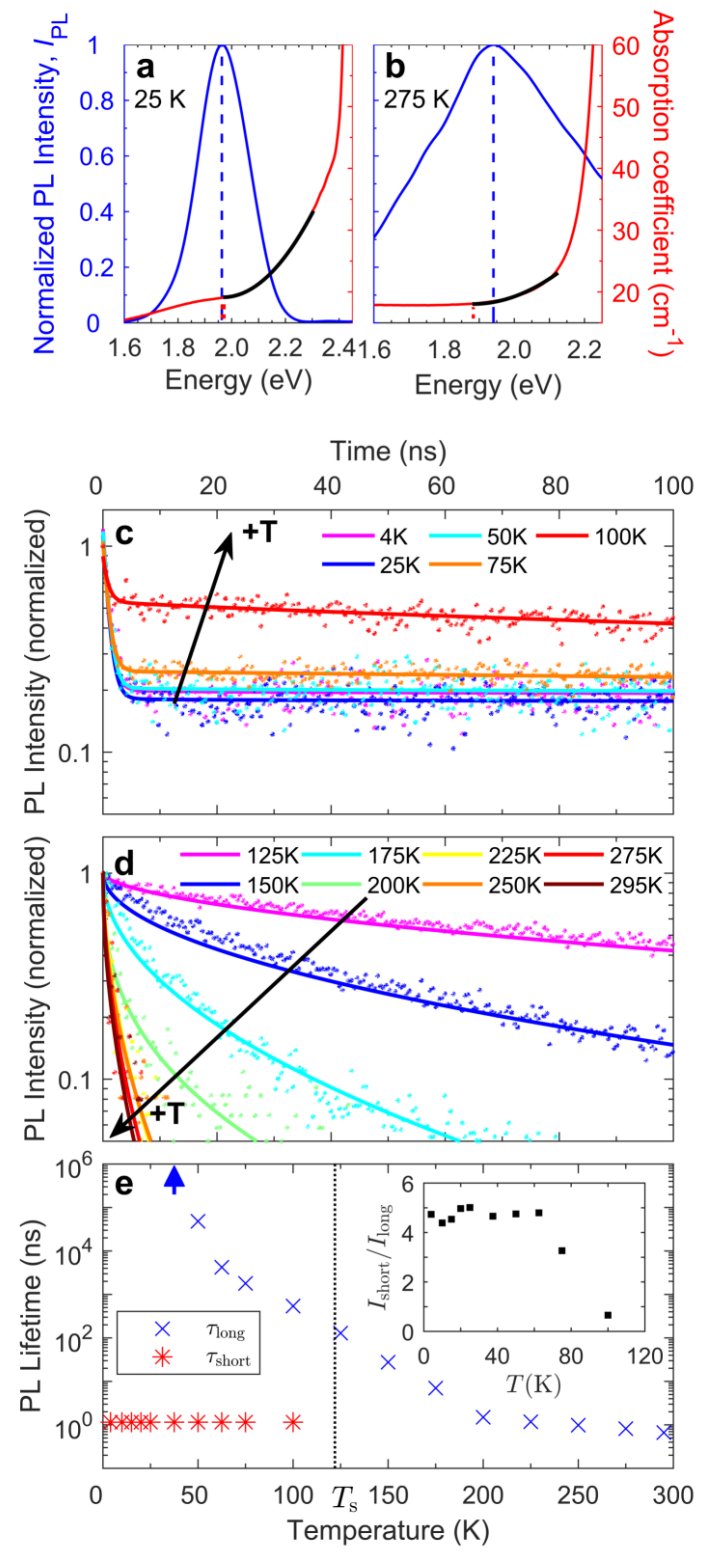

Figure 3: Photoluminescence and absorbance measurements. Steady-state PL (solid blue line) and absorption coefficient (solid red line) at $25 \mathrm{~K}$ (a) and $275 \mathrm{~K}$ (b) as a function of photon energy E. Quadratic fits $\left(\sim\left(\mathrm{E}_{-} \mathrm{E}_{g}^{i}\right)^{2}\right)$ to the absorption onsets are plotted in black, with the minimum energy $\left(\mathrm{E}_{g}^{i}\right)$ indicated by dotted red lines. The dashed blue lines indicate the energy at the maximum PL intensity. (c) Dots: PL transients (excitation at $398 \mathrm{~nm}$, fluence $210 \mathrm{~nJ} \mathrm{~cm}{ }^{-2}$ ) at temperatures between 4 and $100 \mathrm{~K}$ (tetragonal phase). Solid lines: global fits of the sum of a stretched exponential (long) and a monoexponential (short) component, with the lifetime of the latter common between the temperatures, (d)Dots: PL transients at temperatures from 125 to $295 \mathrm{~K}$ (cubic phase) Solid lines: stretched exponential fits. Black arrows indicate the overall temperature trends. (e) Lifetimes of the long (blue) and short (red) components, shown on both sides of the phase transition temperature $T_{\mathrm{s}}$. Below $50 \mathrm{~K}$, the fitted long component lifetimes exceed the measurement capability of the system, as indicated by the blue arrow. The inset shows the temperature dependence of the ratio between the intensities of the short and long components. 
to promote the formation of a single tetragonal domain.

In Figure 3 (panels $\mathbf{c}$ and $\mathbf{d}$ ) we are only fitting the early time decay (up to 100ns), for approximately the first order of magnitude reduction in PL signal. In the SI, we show a transient PL decay for a $\mathrm{Cs}_{2} \mathrm{AgBiBr}_{6}$ crystal, measured at room temperature over a longer time-period (Figure S6). Here it is indeed apparent that there exists a much slower longer component to the PL decay at room temperature (in agreement with the literature ${ }^{9}$ ). Therefore, it appears that there are 3 component to the PL dynamics. The very fast short decay S, at low temperature, which we interpret to be governed by charge trapping at twinning defects. The longer time decay L, at intermediate temperatures, which is sped up considerably as the temperature increases, likely to be due to charge trapping where the required emission of phonons becomes faster as the temperature is raised. The third component is the slow decay visible in the room temperature PL dynamics at long times, which may be governed by the trap depopulation time, rather than the lifetime of the free carrier.

We show further evidence of the influence of the phase transition on the electronic behaviour from steady-state PL measurements excited with constant intensity 532nm radiation $\left(E_{p h}=2.33 \mathrm{eV}\right)$ (Figure 4). Using low intensity CW excitation at 532nm, below the direct band gap, the excitation region is considerably deeper thus sampling a much greater region of the crystal. Under these conditions the intensity of the emission around $1.9 \mathrm{eV}$ is particularly sensitive to temperature, showing a very pronounced maximum just above the tetragonal to cubic transition. Considering going from room temperature down to low temperature, this observation may be due to the non-radiative trapping slowing down with reducing temperature, which would be expected to lead to an increased fraction of radiative emission, as usually observed in lead halide perovskites. ${ }^{27}$ The sudden drop in PL intensity upon further reducing the temperature below the cubic to tetragonal phase transition, is consistent with an large increase in strain induced electronic defects.

In conclusion, our measurements and analysis demonstrate a strong link between the 


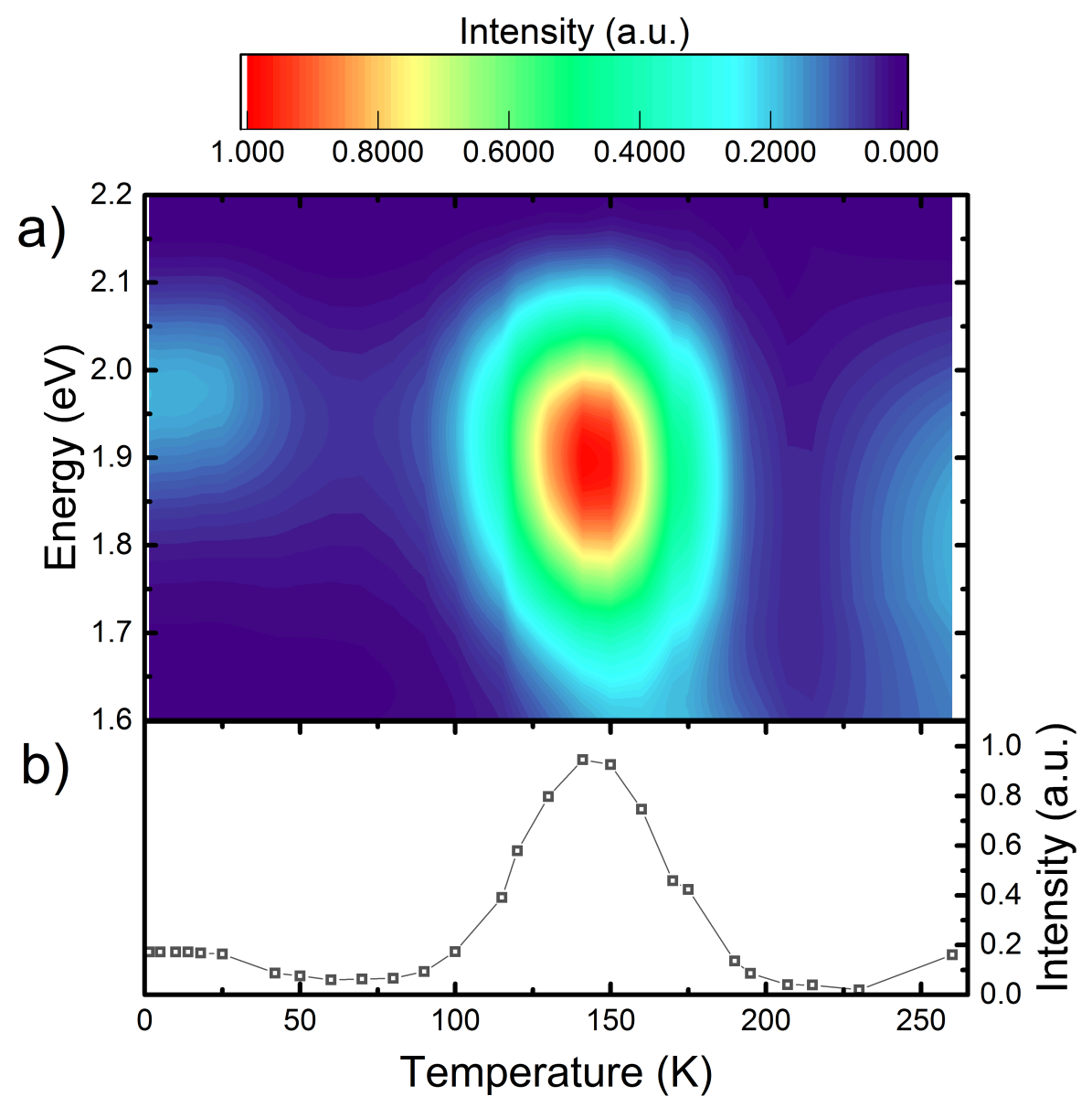

Figure 4: a) Temperature-dependent steady-state PL excited by a $532 \mathrm{~nm}$ CW laser with a constant excitation intensity of $0.05 \mathrm{~W} \mathrm{~cm}^{-2}$. b) Cut of panel $a$ at an energy of $\sim 1.912 \mathrm{eV}$, near the indirect band gap.

structural and optical properties of the double perovskite $\mathrm{Cs}_{2} \mathrm{AgBiBr}_{6}$. Using heat capacity and diffraction measurements, we determined the presence of a structural phase transition at $\sim 122 \mathrm{~K}$, between a room-temperature cubic phase (space group $F m \overline{3} m$ ) and a lowtemperature tetragonal phase (space group $I 4 / m$ ). This phase transition affects the optical properties quite dramatically: the exciton energy, and the corresponding band gap energy, extracted from our reflectance measurement, increases in the tetragonal phase by as much as $20 \mathrm{meV}$, and the excess energy scales linearly with the tetragonal strain, strongly suggesting that the direct gap energy is controlled by the $M \mathrm{Br}_{6}$ octahedral rotation. Moreover, our timeresolved PL measurements demonstrate that the charge carriers lifetimes are also affected 
by the structural transition, with a fast relaxation channel being present mainly in the tetragonal phase, possibly associated with tetragonal twin boundaries. $\mathrm{Cs}_{2} \mathrm{AgBiBr}_{6}$ based solar cells show poorer photo-current and photo-voltage compared to the $\mathrm{Pb}(\mathrm{Sn})$-halide based solar cells ${ }^{11}$ possibly due to the trapping of charge carriers and subsequent non-radiative recombination. Our findings suggest that the formation of twinning boundaries can reduce the charge carrier lifetime. It is likely, therefore, that grain boundaries in polycrystalline thin films could also affect the charge carrier behaviour. As a consequence further device improvement could be expected via better management of grain boundaries.

\section{Acknowledgement}

Experiments at the ISIS Pulsed Neutron and Muon Source were supported by a beamtime allocation from the Science and Technology Facilities Council under the Xpress programme. We acknowledge Ron Smith for support for these experiments. We acknowledge financial support from the UK Engineering and Physical Sciences Research Council (EPSRC) and from Balliol college at Oxford University (J.T. Hamilton scholarship). The work was funded by EPSRC grant No. EP/P033229/1, entitled "Unravelling halide segregation in hybrid perovskites for Si tandem photovoltaics".

\section{Supporting Information Available}

The following files are available free of charge.

- Plot of $\mathrm{F}_{\text {calc }}^{2}$ versus $\mathrm{F}_{\text {obs }}^{2}$ for the structural refinement of a $\mathrm{Cs}_{2} \mathrm{AgBiBr}_{6}$ single crystal and crystal structure (Figure S1 and Table S1);

- Photograph of a $\mathrm{Cs}_{2} \mathrm{AgBiBr}_{6}$ single crystal and real-space representation of the tetragonal phase (Figure S2); 
- Neutron-diffraction patterns with Rietveld refinement for the diffraction profiles measured for $\mathrm{Cs}_{2} \mathrm{AgBiBr}_{6}$ polycrystalline powder (Figure $\mathrm{S} 3$ );

- X-ray diffraction peak splitting (Figure S4);

- Specific heat capacity near the transition edge (Figure S5);

- Modelling of the transition energy (paragraph 3);

- Room temperature time-resolved photoluminescence of a $\mathrm{Cs}_{2} \mathrm{AgBiBr}_{6}$ single crystal on larger time-scale (Figure S6);

- Reflectivity measurements analysis (section 5);

- Experimental details (section 6). 


\section{References}

(1) National Renewable Energy Laboratory (NREL). https://www.nrel.gov/pv/assets/pdfs/pvefficiency-chart.20181214.pdf.

(2) Snaith, H. J. Perovskites: The Emergence of a New Era for Low-Cost, High-Efficiency Solar Cells. J. Phys. Chem. Lett. 2013, 4, 3623-3630.

(3) Lee, M. M.; Teuscher, J.; Miyasaka, T.; Murakami, T. N.; Snaith, H. J. Efficient Hybrid Solar Cells Based on Meso-Superstructured Organometal Halide Perovskites. Science 2012, 338, 643-647.

(4) Koh, T. M.; Fu, K.; Fang, Y.; Chen, S.; Sum, T. C.; Mathews, N.; Mhaisalkar, S. G.; Boix, P. P.; Baikie, T. Formamidinium-Containing Metal-Halide: An Alternative Material for Near-IR Absorption Perovskite Solar Cells. J. Phys. Chem. C 2014, 118, $16458-16462$.

(5) Lee, J.-W.; Seol, D.-J.; Cho, A.-N.; Park, N.-G. High-Efficiency Perovskite Solar Cells Based on the Black Polymorph of $\mathrm{HC}\left(\mathrm{NH}_{2}\right)_{2} \mathrm{PbI}_{3}$. Adv. Mater. 2014, 26, 4991-4998.

(6) Eperon, G. E.; Paternò, G. M.; Sutton, R. J.; Zampetti, A.; Haghighirad, A. A.; Cacialli, F.; Snaith, H. J. Inorganic Caesium Lead Iodide Perovskite Solar Cells. J. Mater. Chem. A 2015, 3, 19688-19695.

(7) Wu, C.; Zhang, Q.; Liu, Y.; Luo, W.; Guo, X.; Huang, Z.; Ting, H.; Sun, W.; Zhong, X.; Wei, S., et al. The Dawn of Lead-Free Perovskite Solar Cell: Highly Stable Double Perovskite $\mathrm{Cs}_{2} \mathrm{AgBiBr}_{6}$ Film. Adv. Sci. (Weinheim, Ger.) 2018, 5, 1700759.

(8) Volonakis, G.; Filip, M. R.; Haghighirad, A. A.; Sakai, N.; Wenger, B.; Snaith, H. J.; Giustino, F. Lead-Free Halide Double Perovskites via Heterovalent Substitution of Noble Metals. J. Phys. Chem. Lett. 2016, \%, 1254-1259. 
(9) Slavney, A. H.; Hu, T.; Lindenberg, A. M.; Karunadasa, H. I. A Bismuth-Halide Double Perovskite with Long Carrier Recombination Lifetime for Photovoltaic Applications. J. Am. Chem. Soc. 2016, 138, 2138-2141.

(10) Greul, E.; Petrus, M. L.; Binek, A.; Docampo, P.; Bein, T. Highly Stable, Phase Pure $\mathrm{Cs}_{2} \mathrm{AgBiBr}_{6}$ Double Perovskite Thin Films for Optoelectronic Applications. J. Mater. Chem. A 2017, 19972-19981.

(11) Gao, W.; Ran, C.; Xi, J.; Jiao, B.; Zhang, W.; Wu, M.; Hou, X.; Wu, Z. High-Quality $\mathrm{Cs}_{2} \mathrm{AgBiBr}_{6}$ Double Perovskite Film for Lead-Free Inverted Planar Heterojunction Solar Cells with 2.2\% Efficiency. ChemPhysChem 2018, 1-6.

(12) Luo, J.; Wang, X.; Li, S.; Liu, J.; Guo, Y.; Niu, G.; Yao, L.; Fu, Y.; Gao, L.; Dong, Q., et al. Efficient and Stable Emission of Warm-White Light from Lead-Free Halide Double Perovskites. Nature 2018, 563, 541-545.

(13) McClure, E. T.; Ball, M. R.; Windl, W.; Woodward, P. M. $\mathrm{Cs}_{2} \operatorname{AgBiX}_{6}(\mathrm{X}=\mathrm{Br}, \mathrm{Cl})$ : New Visible Light Absorbing, Lead-Free Halide Perovskite Semiconductors. Chem. Mater. 2016, 28, 1348-1354.

(14) Filip, M. R.; Hillman, S.; Haghighirad, A. A.; Snaith, H. J.; Giustino, F. Band Gaps of the Lead-Free Halide Double Perovskites $\mathrm{Cs}_{2} \mathrm{AgBiCl}_{6}$ and $\mathrm{Cs}_{2} \mathrm{AgBiBr}_{6}$ from Theory and Experiment. J. Phys. Chem. Lett. 2016, 7, 2579-2585.

(15) Hoye, R. L. Z.; Eyre, L.; Wei, F.; Brivio, F.; Sadhanala, A.; Sun, S.; Li, W.; Zhang, K. H. L.; MacManus-Driscoll, J. L.; Bristowe, P. D., et al. Fundamental Carrier Lifetime Exceeding 1 us in $\mathrm{Cs}_{2} \mathrm{AgBiBr}_{6}$ Double Perovskite. Adv. Mater. Interfaces 2018, 1800464.

(16) Bartesaghi, D.; Slavney, A. H.; Gélvez-Rueda, M. C.; Connor, B. A.; Grozema, F. C.; Karunadasa, H. I.; Savenije, T. J. Charge Carrier Dynamics in $\mathrm{Cs}_{2} \mathrm{AgBiBr}_{6}$ Double Perovskite. J. Phys. Chem. C 2018, 122, 4809-4816. 
(17) Creutz, S. E.; Crites, E. N.; De Siena, M. C.; Gamelin, D. R. Colloidal Nanocrystals of Lead-Free Double-Perovskite (Elpasolite) Semiconductors: Synthesis and Anion Exchange To Access New Materials. Nano Lett. 2018, 18, 1118-1123.

(18) Stoumpos, C. C.; Malliakas, C. D.; Kanatzidis, M. G. Semiconducting Tin and Lead Iodide Perovskites with Organic Cations: Phase Transitions, High Mobilities, and NearInfrared Photoluminescent Properties. Inorg. Chem. 2013, 52, 9019-9038.

(19) Yang, J.; Zhang, P.; Wei, S.-H. Band Structure Engineering of $\mathrm{Cs}_{2} \mathrm{AgBiBr}_{6}$ Perovskite through Order-Disordered Transition: A First-Principle Study. J. Phys. Chem. Lett. 2018, 9, 31-35.

(20) Feng, J. Mechanical Properties of Hybrid Organic-Inorganic $\mathrm{CH}_{3} \mathrm{NH}_{3} \mathrm{BX}_{3}(\mathrm{~B}=\mathrm{Sn}, \mathrm{Pb}$; $\mathrm{X}=\mathrm{Br}, \mathrm{I})$ Perovskites for Solar Cell Absorbers. APL Mater. 2014, 2, 081801.

(21) Rodríguez-Carvajal, J. Recent Advances in Magnetic Structure Determination by Neutron Powder Diffraction. Phys. B (Amsterdam; Neth.) 1993, 192, 55-69.

(22) Stoumpos, C. C.; Kanatzidis, M. G. The Renaissance of Halide Perovskites and Their Evolution as Emerging Semiconductors. Acc. Chem. Res. 2015, 48, 2791-2802.

(23) Knutson, J. L.; Martin, J. D. Tuning the Band Gap in Hybrid Tin Iodide Perovskite Semiconductors Using Structural Templating. Inorg. Chem. 2005, 44, 4699-4705.

(24) We performed a fit of a quadratic function directly to the absorption data, as explained by $\mathrm{Yu}$ and Cadorna ${ }^{28}$ and as previously done in the case of $\mathrm{MAPbI}_{3}$ by Kirchartz et $a l .29$ and Wang et al.. $\stackrel{30}{20}$

(25) Steele, J. A.; Puech, P.; Keshavarz, M.; Yang, R.; Banerjee, S.; Debroye, E.; Kim, C. W.; Yuan, H.; Heo, N. H.; Vanacken, J., et al. Giant Electron-Phonon Coupling and Deep Conduction Band Resonance in Metal Halide Double Perovskite. ACS Nano 2018, 12, $8081-8090$. 
(26) D’Innocenzo, V.; Grancini, G.; Alcocer, M. J. P.; Kandada, A. R. S.; Stranks, S. D.; Lee, M. M.; Lanzani, G.; Snaith, H. J.; Petrozza, A. Excitons Versus Free Charges in Organo-Lead Tri-Halide Perovskites. Nat. Commun. 2014, 5, 3586.

(27) Stranks, S. D.; Burlakov, V. M.; Leijtens, T.; Ball, J. M.; Goriely, A.; Snaith, H. J. Recombination Kinetics in Organic-Inorganic Perovskites: Excitons, Free Charge, and Subgap States. Phys. Rev. Appl. 2014, 2, 034007.

(28) Yu, P. Y.; Cardona, M. Fundamentals of Semiconductors: Physics and Materials Properties.; Graduate Texts in Physics; Springer Berlin Heidelberg: Berlin, Heidelberg, 2010; Vol. 1; pp 1829-1841.

(29) Kirchartz, T.; Rau, U. Decreasing Radiative Recombination Coefficients via an Indirect Band Gap in Lead Halide Perovskites. J. Phys. Chem. Lett. 2017, 8, 1265-1271.

(30) Wang, T.; Daiber, B.; Frost, J. M.; Mann, S. A.; Garnett, E. C.; Walsh, A.; Ehrler, B. Indirect to Direct Bandgap Transition in Methylammonium Lead Halide Perovskite. Energy Environ. Sci. 2017, 10, 509-515. 